\title{
On the General Solution of Impulsive Systems with Hadamard Fractional Derivatives
}

\author{
Xianmin Zhang, ${ }^{1}$ Xianzhen Zhang, ${ }^{2}$ Zuohua Liu, ${ }^{3}$ Wenbin Ding, \\ Hui Cao, ${ }^{1}$ and Tong Shu ${ }^{1}$ \\ ${ }^{1}$ School of Electronic Engineering, Jiujiang University, Jiujiang, Jiangxi 332005, China \\ ${ }^{2}$ School of Chemical and Environmental Engineering, Jiujiang University, Jiujiang, Jiangxi 332005, China \\ ${ }^{3}$ School of Chemistry and Chemical Engineering, Chongqing University, Chongqing 400044, China
}

Correspondence should be addressed to Xianmin Zhang; z6x2m@126.com

Received 20 January 2016; Accepted 8 May 2016

Academic Editor: Josè A. Tenereiro Machado

Copyright (C) 2016 Xianmin Zhang et al. This is an open access article distributed under the Creative Commons Attribution License, which permits unrestricted use, distribution, and reproduction in any medium, provided the original work is properly cited.

This paper is concerned with the solution for impulsive differential equations with Hadamard fractional derivatives. The general solution of this impulsive fractional system is found by considering the limit case in which impulses approach zero. Next, an example is provided to expound the theoretical result.

\section{Introduction}

The theory of fractional calculus has been applied in widespread fields of science and engineering [1-3], and some properties of the solution were researched for fractional differential equations in [4-11]. Moreover, the Hadamard-type fractional calculus which is a branch of fractional calculus was developed in [12-17]. Next, the Caputo-Hadamard fractional derivative which is a Caputo-type modification of the Hadamard fractional derivative is suggested in [18], some fundamental theorems of this fractional derivative were proved in [19], and Cauchy problems of a differential equation with a left Caputo-Hadamard fractional derivative were studied in spaces of continuously differentiable functions in [20].

On the other hand, impulsive differential equations are often used for description some processes or system with impulsive effects, impulsive (partial) differential equations with Caputo fractional derivative were widely studied in [21-32], and the existence of solutions was considered for impulsive differential equations with Hadamard fractional derivative in [33].
Furthermore, the general solution for some impulsive fractional differential equations was found in [34-39]. Therefore, we will seek the general solution for the impulsive systems with Hadamard fractional derivatives in present paper:

$$
\begin{aligned}
{ }_{H} D_{a^{+}}^{q} z(t) & =g(t, z(t)), \\
& t \in[a, T], t \neq t_{i}(i=1,2, \ldots, m), \\
\left.\Delta\left({ }_{H}{ }^{\mathcal{f}_{a^{+}}^{1-q}} z\right)\right|_{t=t_{i}} & ={ }_{H^{f}}{ }_{a^{+}}^{1-q} z\left(t_{i}^{+}\right)-{ }_{H^{f^{+}}}{ }_{a^{+}}^{1-q} z\left(t_{i}^{-}\right) \\
& =J_{i}\left(z\left(t_{i}^{-}\right)\right) \in \mathbb{C}, \quad i=1,2, \ldots, m, \\
{ }_{H}{ }^{\mathcal{F}_{a^{+}}^{1-q}} z\left(a^{+}\right) & =z_{a}, \quad z_{a} \in \mathbb{C},
\end{aligned}
$$

where $q \in \mathbb{C}$ and $\Re(q) \in(0,1),{ }_{H} D_{a^{+}}^{q}$ denotes left-sided Hadamard fractional derivative of order $q$ and $a>0$, and $H_{a^{+}}^{\mathscr{f}^{1-q}}$ denotes left-sided Hadamard fractional integral of order $1-q \cdot g:[a, T] \times \mathbb{C} \rightarrow \mathbb{C}$ and $J_{i}: \mathbb{C} \rightarrow \mathbb{C}(i=1, \ldots, m)$ are some appropriate functions and impulsive points $a=t_{0}<t_{1}<\cdots<$ $t_{m}<t_{m+1}=T .{ }_{H} \mathcal{F}_{a^{+}}^{1-q} z\left(t_{i}^{+}\right)=\lim _{\varepsilon \rightarrow 0^{+}}{ }_{H}{ }_{a^{+}}^{1-q} z\left(t_{i}+\varepsilon\right)$ and 
$H^{\mathcal{J}_{a^{+}}^{1-q}} z\left(t_{i}^{-}\right)=\lim _{\varepsilon \rightarrow 0^{-}} \mathcal{F}_{a^{+}}^{1-q} z\left(t_{i}+\varepsilon\right)$ represent the right and left limits of ${ }_{H^{J^{2}}}^{\mathscr{F}^{1-q}} z(t)$ at $t=t_{i}$, respectively.
Considering limited case in system (1a)-(1c), we have

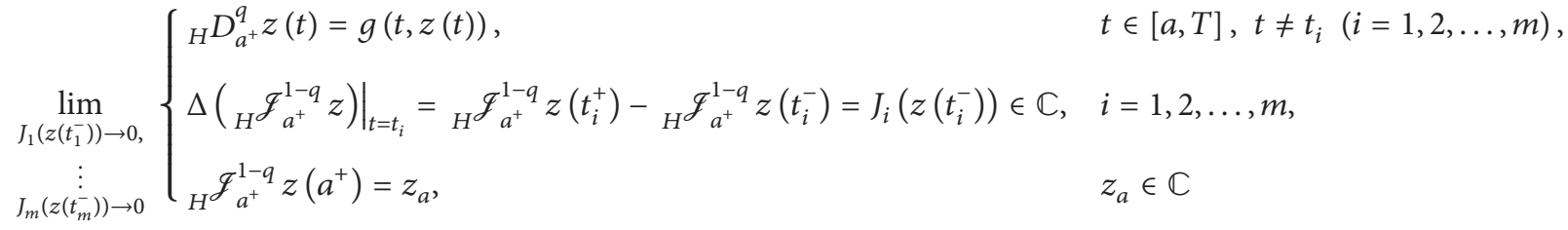

$$
\begin{aligned}
& = \begin{cases}{ }_{H} D_{a^{+}}^{q} z(t)=f(t, z(t)), & t \in(a, T], \\
{ }_{H^{J^{2}}}{ }_{a^{+}}^{1-q} z(a)=z_{a}, & z_{a} \in \mathbb{C} .\end{cases}
\end{aligned}
$$

Therefore,

$$
\begin{aligned}
& \lim _{J_{1}\left(z\left(t_{1}^{-}\right)\right) \rightarrow 0,}\{\text { the solution of impulsive system (1a) }-(1 \mathrm{c})\} \\
& \vdots \\
& J_{m}\left(z\left(t_{m}^{-}\right)\right) \rightarrow 0
\end{aligned}
$$$$
=\{\text { the solution of system }(2)\} \text {. }
$$

Next, using the definition of Hadamard fractional derivative, system (1a)-(1c) is transformed into

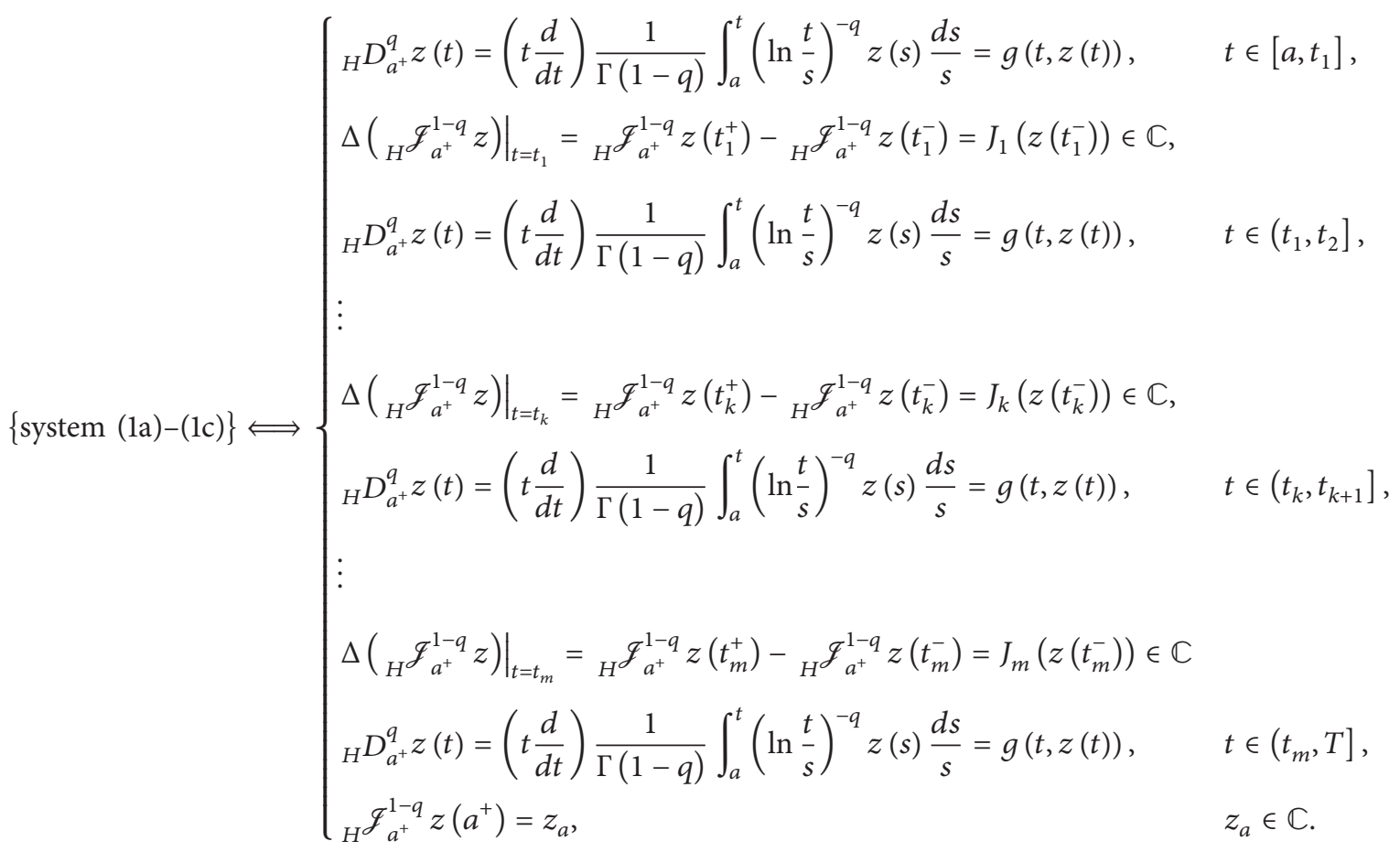

According to each interval $\left(t_{k}, t_{k+1}\right]$ (here $k=0,1,2, \ldots, m$ ) in (4), define a function

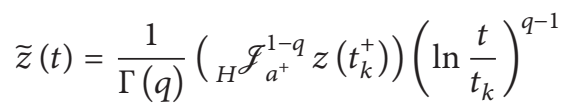

$$
+\frac{1}{\Gamma(q)} \int_{t_{k}}^{t}\left(\ln \frac{t}{s}\right)^{q-1} g(s, z(s)) \frac{d s}{s},
$$$$
\text { for } t \in\left(t_{k}, t_{k+1}\right] \text {, }
$$

with ${ }_{H^{J}} \mathscr{J}_{a^{+}}^{1-q} z\left(t_{k}^{+}\right)={ }_{H^{J^{*}}}^{\mathscr{F}_{a^{+}}^{1-q}} z\left(t_{k}^{-}\right)+J_{k}\left(z\left(t_{k}^{-}\right)\right)$. 
By Definition 2, we have

$$
\begin{aligned}
& {\left[{ }_{H} D_{a^{+}}^{q} \widetilde{z}(t)\right]_{t \in\left(t_{k}, t_{k+1}\right]}={ }_{H} D_{a^{+}}^{q}\left\{\frac{1}{\Gamma(q)}\left({ }_{H}{ }_{a^{+}}^{1-q} z\left(t_{k}^{+}\right)\right)\right.} \\
& \left.\cdot\left(\ln \frac{t}{t_{k}}\right)^{q-1}+\frac{1}{\Gamma(q)} \int_{t_{k}}^{t}\left(\ln \frac{t}{s}\right)^{q-1} g(s, z(s)) \frac{d s}{s}\right\} \\
& =\frac{1}{\Gamma(q) \Gamma(1-q)}\left(t \frac{d}{d t}\right) \int_{t_{k}}^{t}\left(\ln \frac{t}{\eta}\right)^{1-q-1} \\
& \cdot\left[\int_{t_{k}}^{\eta}\left(\ln \frac{\eta}{s}\right)^{q-1} g(s, z(s)) \frac{d s}{s}\right] \frac{d \eta}{\eta} \\
& =\left.g(t, z(t))\right|_{t \in\left(t_{k}, t_{k+1}\right]} .
\end{aligned}
$$

Therefore, $\widetilde{z}(t)$ satisfies condition (1a), and $\widetilde{z}(t)$ also satisfies conditions (1b) and (1c). But $\widetilde{z}(t)$ does not satisfy condition (3). Thus, $\widetilde{z}(t)$ is not a solution of system (1a)-(1c) and will be considered as an approximate solution to seek the exact solution of system (1a)-(1c).

Next, we will give some definitions and conclusions in Section 2 and prove the formula of general solution for impulsive differential equations with Hadamard fractional derivative in Section 3. Finally, we will provide an example to expound the main result in Section 4.

\section{Preliminaries}

Definition 1 (see [2]). Let $0 \leq a \leq T \leq \infty$ be finite or infinite interval of the half-axis $\mathbb{R}^{+}$. The left-sided Hadamard fractional integral of order $\alpha \in \mathbb{C}(\mathfrak{R}(\alpha)>0)$ of function $\varphi(x)$ is defined by

$$
\begin{aligned}
\left({ }_{H} \mathcal{F}_{a^{+}}^{\alpha} \varphi\right)(x)=\frac{1}{\Gamma(\alpha)} \int_{a}^{x}\left(\ln \frac{x}{s}\right)^{\alpha-1} \varphi(s) \frac{d s}{s}, & \\
& (a<x<T),
\end{aligned}
$$

where $\Gamma(\cdot)$ is the Gamma function.
Definition 2 (see [2]). The left-sided Hadamard fractional derivative of order $\alpha \in \mathbb{C}$ with $\mathfrak{R}(\alpha) \geq 0$ on $(a, T)$ is defined by

$$
\begin{aligned}
&\left({ }_{H} D_{a^{+}}^{\alpha} \varphi\right)(x)=\delta^{n}\left({ }_{H} \mathcal{J}_{a^{+}}^{n-\alpha} \varphi\right)(x) \\
&=\left(x \frac{d}{d x}\right)^{n} \frac{1}{\Gamma(n-\alpha)} \int_{a}^{x}\left(\ln \frac{x}{s}\right)^{n-\alpha-1} \varphi(s) \frac{d s}{s}, \\
& \quad(a<x<T),
\end{aligned}
$$

where $n=[\Re(\alpha)]+1$ and differential operator $\delta=x(d / d x)$ with $\delta^{0} y(x)=y(x)$.

Lemma 3 (see [2]). Let $\mathfrak{R}(\alpha) \geq 0$ and $n=[\Re(\alpha)]+1,0<$ $a<T<\infty$. The fractional Cauchy problem

$$
\begin{aligned}
{ }_{H} D_{a^{+}}^{\alpha} y(x) & =h(x, y(x)), \quad x \in(a, T], \\
{ }_{H} D_{a^{+}}^{\alpha-i} y\left(a^{+}\right) & =b_{i} \in \mathbb{C}, \quad i=1,2, \ldots, n
\end{aligned}
$$

is equivalent to the following nonlinear integral equation:

$$
\begin{aligned}
y(x)= & \sum_{i=1}^{n} \frac{b_{i}}{\Gamma(\alpha-i+1)}\left(\ln \frac{x}{a}\right)^{\alpha-i} \\
& +\frac{1}{\Gamma(\alpha)} \int_{a}^{x}\left(\ln \frac{x}{s}\right)^{\alpha-1} h(s, y(s)) \frac{d s}{s}
\end{aligned}
$$

$(0<a<x)$.

\section{Main Result}

Theorem 4. Let $q \in \mathbb{C}, \mathfrak{R}(q) \in(0,1)$, and $\sum_{i=1}^{0} x_{i}=0$, and let $\xi$ be an arbitrary constant. System (1a)-(1c) is equivalent to the integral equation:

$$
\begin{aligned}
& z(t)=\frac{z_{a}}{\Gamma(q)}\left(\ln \frac{t}{a}\right)^{q-1}+\frac{1}{\Gamma(q)} \int_{a}^{t}\left(\ln \frac{t}{s}\right)^{q-1} g(s, z(s)) \frac{d s}{s}+\sum_{i=1}^{k} \frac{J_{i}\left(z\left(t_{i}^{-}\right)\right)}{\Gamma(q)}\left(\ln \frac{t}{t_{i}}\right)^{q-1}-\frac{\xi}{\Gamma(q)} \sum_{i=1}^{k} J_{i}\left(z\left(t_{i}^{-}\right)\right) \\
& .\left\{z_{a}\left(\ln \frac{t}{a}\right)^{q-1}+\int_{a}^{t}\left(\ln \frac{t}{s}\right)^{q-1} g(s, z(s)) \frac{d s}{s}-\left[z_{a}+\int_{a}^{t_{i}} g(s, z(s)) \frac{d s}{s}\right]\left(\ln \frac{t}{t_{i}}\right)^{q-1}-\int_{t_{i}}^{t}\left(\ln \frac{t}{s}\right)^{q-1} g(s, z(s)) \frac{d s}{s}\right\} \\
& \quad \text { for } t \in\left(t_{k}, t_{k+1}\right], k \in\{0,1,2, \ldots, m\}
\end{aligned}
$$

provided that the integral in (11) exists.

Proof.

Necessity. Letting $J_{i}\left(z\left(t_{i}^{-}\right)\right)=0(i=1,2, \ldots, k)$ in (11), we have

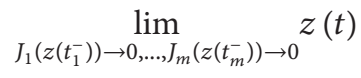

$$
\begin{aligned}
= & \frac{z_{a}}{\Gamma(q)}\left(\ln \frac{t}{a}\right)^{q-1} \\
& +\frac{1}{\Gamma(q)} \int_{a}^{t}\left(\ln \frac{t}{s}\right)^{q-1} g(s, z(s)) \frac{d s}{s}
\end{aligned}
$$$$
\text { for } t \in\left(t_{k}, t_{k+1}\right], k \in\{0,1,2, \ldots, m\} \text {. }
$$ 
4

Mathematical Problems in Engineering

According to Lemma 3, (12) is equivalent to (2). Therefore, (11) satisfies condition (3).

Next, using Definition 2 for (11), we have

$$
\begin{aligned}
& \left.{ }_{H} D_{a^{+}}^{q} z(t)\right|_{t \in\left(t_{k}, t_{k+1}\right]}={ }_{H} D_{a^{+}}^{q}\left\{\frac{z_{a}}{\Gamma(q)}\left(\ln \frac{t}{a}\right)^{q-1}\right. \\
& +\frac{1}{\Gamma(q)} \int_{a}^{t}\left(\ln \frac{t}{s}\right)^{q-1} g(s, z(s)) \frac{d s}{s} \\
& +\sum_{i=1}^{k} \frac{J_{i}\left(z\left(t_{i}^{-}\right)\right)}{\Gamma(q)}\left(\ln \frac{t}{t_{i}}\right)^{q-1}-\frac{\xi}{\Gamma(q)} \sum_{i=1}^{k} J_{i}\left(z\left(t_{i}^{-}\right)\right) \\
& \cdot\left\{z_{a}\left(\ln \frac{t}{a}\right)^{q-1}+\int_{a}^{t}\left(\ln \frac{t}{s}\right)^{q-1} g(s, z(s)) \frac{d s}{s}\right. \\
& -\left[z_{a}+\int_{a}^{t_{i}} g(s, z(s)) \frac{d s}{s}\right]\left(\ln \frac{t}{t_{i}}\right)^{q-1} \\
& \left.\left.-\int_{t_{i}}^{t}\left(\ln \frac{t}{s}\right)^{q-1} g(s, z(s)) \frac{d s}{s}\right\}\right\}=g(t, \\
& z(t))\left._{t \geq a}\right|_{t \in\left(t_{k}, t_{k+1}\right]}-\left\{\sum _ { i = 1 } ^ { k } \xi J _ { i } ( z ( t _ { i } ^ { - } ) ) \left[g(t, z(t))_{t \geq a}\right.\right. \\
& \left.\left.-g(t, z(t))_{t \geq t_{i}}\right]\right\}_{t \in\left(t_{k}, t_{k+1}\right]}=\left.g(t, z(t))\right|_{t \in\left(t_{k}, t_{k+1}\right]} .
\end{aligned}
$$

So (11) satisfies (la).

By (11), we get

$$
\begin{aligned}
& H_{H^{+}}^{\mathcal{J}^{1-q}} z\left(t_{k}^{+}\right)-{ }_{H^{J}} \mathcal{F}_{a^{+}}^{1-q} z\left(t_{k}^{-}\right)=\left\{\frac{1}{\Gamma(1-q)}\right. \\
& \left.\cdot \int_{a}^{t}\left(\ln \frac{t}{\eta}\right)^{1-q-1} z(\eta) \frac{d \eta}{\eta}\right\}_{t \rightarrow t_{k}^{+}}-\left\{\frac{1}{\Gamma(1-q)}\right. \\
& \left.\cdot \int_{a}^{t}\left(\ln \frac{t}{\eta}\right)^{1-q-1} z(\eta) \frac{d \eta}{\eta}\right\}_{t \rightarrow t_{k}^{-}}=\left\{J_{k}\left(z\left(t_{k}^{-}\right)\right)\right. \\
& -\xi J_{k}\left(z\left(t_{k}^{-}\right)\right)\left[z_{a}+\int_{a}^{t} g(s, z(s)) \frac{d s}{s}\right. \\
& -\left(z_{a}+\int_{a}^{t_{k}} g(s, z(s)) \frac{d s}{s}\right) \\
& \left.\left.-\int_{t_{k}}^{t} g(s, z(s)) \frac{d s}{s}\right]\right\}_{t \rightarrow t_{k}^{+}}=J_{k}\left(z\left(t_{k}^{-}\right)\right) .
\end{aligned}
$$

Therefore, (11) satisfies (lb). Thus, (11) satisfies all conditions of system (1a)-(1c).
Sufficiency (by Mathematical Induction). By Lemma 3, the solution of system (1a)-(1c) satisfies

$$
\begin{aligned}
z(t)= & \frac{z_{a}}{\Gamma(q)}\left(\ln \frac{t}{a}\right)^{q-1} \\
& +\frac{1}{\Gamma(q)} \int_{a}^{t}\left(\ln \frac{t}{s}\right)^{q-1} g(s, z(s)) \frac{d s}{s}
\end{aligned}
$$

for $t \in\left(a, t_{1}\right]$.

Using (15), we obtain

$$
\begin{aligned}
H_{a^{+}}^{\mathcal{F}^{1-q}} z\left(t_{1}^{+}\right) & ={ }_{H^{\mathcal{J}^{+}}}{ }_{a^{+}}^{1-q} z\left(t_{1}^{-}\right)+J_{1}\left(z\left(t_{1}^{-}\right)\right) \\
& =z_{a}+J_{1}\left(z\left(t_{1}^{-}\right)\right)+\int_{a}^{t_{1}} g(s, z(s)) \frac{d s}{s} .
\end{aligned}
$$

Therefore, the approximate solution $\widetilde{z}(t)$ is provided by

$$
\begin{aligned}
& \widetilde{z}(t)=\frac{1}{\Gamma(q)}\left({ }_{H^{\mathcal{J}^{+}}}{ }_{a^{+}}^{1-q} z\left(t_{1}^{+}\right)\right)\left(\ln \frac{t}{t_{1}}\right)^{q-1}+\frac{1}{\Gamma(q)} \\
& \cdot \int_{t_{1}}^{t}\left(\ln \frac{t}{s}\right)^{q-1} g(s, z(s)) \frac{d s}{s} \\
& =\frac{1}{\Gamma(q)}\left[z_{a}+J_{1}\left(z\left(t_{1}^{-}\right)\right)+\int_{a}^{t_{1}} g(s, z(s)) \frac{d s}{s}\right] \\
& \cdot\left(\ln \frac{t}{t_{1}}\right)^{q-1}+\frac{1}{\Gamma(q)} \int_{t_{1}}^{t}\left(\ln \frac{t}{s}\right)^{q-1} g(s, z(s)) \frac{d s}{s} \\
& \text { for } t \in\left(t_{1}, t_{2}\right] \text {. }
\end{aligned}
$$

Let $e_{1}(t)=z(t)-\widetilde{z}(t)$ for $t \in\left(t_{1}, t_{2}\right]$. Moreover, by (15), the exact solution $z(t)$ of system (1a)-(1c) satisfies

$$
\begin{aligned}
\lim _{J_{1}\left(z\left(t_{1}^{-}\right)\right) \rightarrow 0} z(t)= & \frac{z_{a}}{\Gamma(q)}\left(\ln \frac{t}{a}\right)^{q-1} \\
& +\frac{1}{\Gamma(q)} \int_{a}^{t}\left(\ln \frac{t}{s}\right)^{q-1} g(s, z(s)) \frac{d s}{s}, \\
& \text { for } t \in\left(t_{1}, t_{2}\right] .
\end{aligned}
$$

Therefore,

$$
\begin{aligned}
\lim _{J_{1}\left(z\left(t_{1}^{-}\right)\right) \rightarrow 0} e_{1}(t)=\lim _{J_{1}\left(z\left(t_{1}^{-}\right)\right) \rightarrow 0}\{z(t)-\widetilde{z}(t)\} \\
=\frac{1}{\Gamma(q)}\left\{z_{a}\left(\ln \frac{t}{a}\right)^{q-1}\right. \\
+\int_{a}^{t}\left(\ln \frac{t}{s}\right)^{q-1} g(s, z(s)) \frac{d s}{s} \\
-\left[z_{a}+\int_{a}^{t_{1}} g(s, z(s)) \frac{d s}{s}\right]\left(\ln \frac{t}{t_{1}}\right)^{q-1} \\
\left.-\int_{t_{1}}^{t}\left(\ln \frac{t}{s}\right)^{q-1} g(s, z(s)) \frac{d s}{s}\right\} .
\end{aligned}
$$


Mathematical Problems in Engineering

5

By (19), let

$$
\begin{aligned}
e_{1}(t) & =\sigma\left[J_{1}\left(z\left(t_{1}^{-}\right)\right)\right] \lim _{J_{1}\left(z\left(t_{1}^{-}\right)\right) \rightarrow 0} e_{1}(t) \\
= & \frac{\sigma\left[J_{1}\left(z\left(t_{1}^{-}\right)\right)\right]}{\Gamma(q)}\left\{z_{a}\left(\ln \frac{t}{a}\right)^{q-1}\right. \\
+ & \int_{a}^{t}\left(\ln \frac{t}{s}\right)^{q-1} g(s, z(s)) \frac{d s}{s} \\
- & {\left[z_{a}+\int_{a}^{t_{1}} g(s, z(s)) \frac{d s}{s}\right]\left(\ln \frac{t}{t_{1}}\right)^{q-1} } \\
- & \left.\int_{t_{1}}^{t}\left(\ln \frac{t}{s}\right)^{q-1} g(s, z(s)) \frac{d s}{s}\right\},
\end{aligned}
$$

where $\sigma(\cdot)$ is an undetermined function with $\sigma(0)=1$. Thus,

$$
\begin{gathered}
z(t)=\widetilde{z}(t)+e_{1}(t)=\frac{\sigma\left[J_{1}\left(z\left(t_{1}^{-}\right)\right)\right]}{\Gamma(q)}\left[z_{a}\left(\ln \frac{t}{a}\right)^{q-1}\right. \\
\left.+\int_{a}^{t}\left(\ln \frac{t}{s}\right)^{q-1} g(s, z(s)) \frac{d s}{s}\right] \\
+\frac{J_{1}\left(z\left(t_{1}^{-}\right)\right)}{\Gamma(q)}\left(\ln \frac{t}{t_{1}}\right)^{q-1} \\
+\frac{1-\sigma\left[J_{1}\left(z\left(t_{1}^{-}\right)\right)\right]}{\Gamma(q)}\left\{\left[z_{a}+\int_{a}^{t_{1}} g(s, z(s)) \frac{d s}{s}\right]\right. \\
\left..\left(\ln \frac{t}{t_{1}}\right)^{q-1}+\int_{t_{1}}^{t}\left(\ln \frac{t}{s}\right)^{q-1} g(s, z(s)) \frac{d s}{s}\right\} \\
\quad \text { for } t \in\left(t_{1}, t_{2}\right] .
\end{gathered}
$$

Using (21), we get

$$
\begin{aligned}
H^{\mathcal{J}_{a^{+}}^{1-q}} z\left(t_{2}^{+}\right)= & H_{a^{+}}^{\mathcal{J}^{1-q}} z\left(t_{2}^{-}\right)+J_{2}\left(z\left(t_{2}^{-}\right)\right) \\
= & z_{a}+J_{1}\left(z\left(t_{1}^{-}\right)\right)+J_{2}\left(z\left(t_{2}^{-}\right)\right) \\
& +\int_{a}^{t_{2}} g(s, z(s)) \frac{d s}{s} .
\end{aligned}
$$

Then the approximate solution $\widetilde{z}(t)$ is given by

$$
\begin{gathered}
\widetilde{z}(t)=\frac{1}{\Gamma(q)}\left({ }_{H^{\prime}} \mathcal{F}_{a^{+}}^{1-q} z\left(t_{2}^{+}\right)\right)\left(\ln \frac{t}{t_{2}}\right)^{q-1}+\frac{1}{\Gamma(q)} \\
\cdot \int_{t_{2}}^{t}\left(\ln \frac{t}{s}\right)^{q-1} g(s, z(s)) \frac{d s}{s}=\frac{1}{\Gamma(q)}\left[z_{a}\right. \\
\left.+J_{1}\left(z\left(t_{1}^{-}\right)\right)+J_{2}\left(z\left(t_{2}^{-}\right)\right)+\int_{a}^{t_{2}} g(s, z(s)) \frac{d s}{s}\right] \\
\cdot\left(\ln \frac{t}{t_{2}}\right)^{q-1}+\frac{1}{\Gamma(q)} \int_{t_{2}}^{t}\left(\ln \frac{t}{s}\right)^{q-1} g(s, z(s)) \frac{d s}{s} \\
\text { for } t \in\left(t_{2}, t_{3}\right] .
\end{gathered}
$$

Let $e_{2}(t)=z(t)-\widetilde{z}(t)$ for $t \in\left(t_{2}, t_{3}\right]$. By (21), the exact solution $z(t)$ of (1a)-(1c) satisfies

$$
\begin{aligned}
& \lim _{J_{1}\left(z\left(t_{1}^{-}\right)\right) \rightarrow 0} z(t)=\frac{\sigma\left[J_{2}\left(z\left(t_{2}^{-}\right)\right)\right]}{\Gamma(q)}\left[z_{a}\left(\ln \frac{t}{a}\right)^{q-1}\right. \\
& \left.+\int_{a}^{t}\left(\ln \frac{t}{s}\right)^{q-1} g(s, z(s)) \frac{d s}{s}\right] \\
& +\frac{J_{2}\left(z\left(t_{2}^{-}\right)\right)}{\Gamma(q)}\left(\ln \frac{t}{t_{2}}\right)^{q-1} \\
& +\frac{1-\sigma\left[J_{2}\left(z\left(t_{2}^{-}\right)\right)\right]}{\Gamma(q)}\left\{\left[z_{a}+\int_{a}^{t_{2}} g(s, z(s)) \frac{d s}{s}\right]\right.
\end{aligned}
$$

$$
\left.\cdot\left(\ln \frac{t}{t_{2}}\right)^{q-1}+\int_{t_{2}}^{t}\left(\ln \frac{t}{s}\right)^{q-1} g(s, z(s)) \frac{d s}{s}\right\}
$$

$$
\begin{aligned}
& \left.+\int_{a}^{t}\left(\ln \frac{t}{s}\right)^{q-1} g(s, z(s)) \frac{d s}{s}\right] \\
& +\frac{J_{1}\left(z\left(t_{1}^{-}\right)\right)}{\Gamma(q)}\left(\ln \frac{t}{t_{1}}\right)^{q-1} \\
& +\frac{1-\sigma\left[J_{1}\left(z\left(t_{1}^{-}\right)\right)\right]}{\Gamma(q)}\left\{\left[z_{a}+\int_{a}^{t_{1}} g(s, z(s)) \frac{d s}{s}\right]\right. \\
& \left.\cdot\left(\ln \frac{t}{t_{1}}\right)^{q-1}+\int_{t_{1}}^{t}\left(\ln \frac{t}{s}\right)^{q-1} g(s, z(s)) \frac{d s}{s}\right\} \\
& \text { for } t \in\left(t_{2}, t_{3}\right],
\end{aligned}
$$

$$
\begin{aligned}
& \lim _{\substack{J_{1}\left(z\left(t_{1}^{-}\right)\right) \rightarrow 0, J_{2}\left(z\left(t_{1}^{-}\right)\right) \rightarrow 0}} z(t)=\frac{z_{a}}{\Gamma(q)}\left(\ln \frac{t}{a}\right)^{q-1}+\frac{1}{\Gamma(q)} \\
& \cdot \int_{a}^{t}\left(\ln \frac{t}{s}\right)^{q-1} g(s, z(s)) \frac{d s}{s} \text { for } t \in\left(t_{2}, t_{3}\right] .
\end{aligned}
$$

Therefore,

$$
\begin{gathered}
\lim _{J_{1}\left(z\left(t_{1}^{-}\right)\right) \rightarrow 0} e_{2}(t)=\lim _{J_{1}\left(z\left(t_{1}^{-}\right)\right) \rightarrow 0}\{z(t)-\widetilde{z}(t)\} \\
=\frac{\sigma\left[J_{2}\left(z\left(t_{2}^{-}\right)\right)\right]}{\Gamma(q)}\left[z_{a}\left(\ln \frac{t}{a}\right)^{q-1}\right. \\
+\int_{a}^{t}\left(\ln \frac{t}{s}\right)^{q-1} g(s, z(s)) \frac{d s}{s}
\end{gathered}
$$


6

Mathematical Problems in Engineering

$$
\begin{aligned}
& -\left(z_{a}+\int_{a}^{t_{2}} g(s, z(s)) \frac{d s}{s}\right)\left(\ln \frac{t}{t_{2}}\right)^{q-1} \\
& \left.-\int_{t_{2}}^{t}\left(\ln \frac{t}{s}\right)^{q-1} g(s, z(s)) \frac{d s}{s}\right] \text {, } \\
& \lim _{J_{2}\left(z\left(t_{2}^{-}\right)\right) \rightarrow 0} e_{2}(t)=\lim _{J_{2}\left(z\left(t_{2}^{-}\right)\right) \rightarrow 0}\{z(t)-\widetilde{z}(t)\} \\
& =\frac{\sigma\left[J_{1}\left(z\left(t_{1}^{-}\right)\right)\right]}{\Gamma(q)}\left[z_{a}\left(\ln \frac{t}{a}\right)^{q-1}\right. \\
& \left.+\int_{a}^{t}\left(\ln \frac{t}{s}\right)^{q-1} g(s, z(s)) \frac{d s}{s}\right] \\
& +\frac{J_{1}\left(z\left(t_{1}^{-}\right)\right)}{\Gamma(q)}\left(\ln \frac{t}{t_{1}}\right)^{q-1}-\frac{J_{1}\left(z\left(t_{1}^{-}\right)\right)}{\Gamma(q)}\left(\ln \frac{t}{t_{2}}\right)^{q-1} \\
& +\frac{1-\sigma\left[J_{1}\left(z\left(t_{1}^{-}\right)\right)\right]}{\Gamma(q)}\left\{\left[z_{a}+\int_{a}^{t_{1}} g(s, z(s)) \frac{d s}{s}\right]\right. \\
& \left.\cdot\left(\ln \frac{t}{t_{1}}\right)^{q-1}+\int_{t_{1}}^{t}\left(\ln \frac{t}{s}\right)^{q-1} g(s, z(s)) \frac{d s}{s}\right\} \\
& -\frac{1}{\Gamma(q)}\left[z_{a}+\int_{a}^{t_{2}} g(s, z(s)) \frac{d s}{s}\right]\left(\ln \frac{t}{t_{2}}\right)^{q-1} \\
& -\frac{1}{\Gamma(q)} \int_{t_{2}}^{t}\left(\ln \frac{t}{s}\right)^{q-1} g(s, z(s)) \frac{d s}{s}, \\
& \lim _{\substack{J_{1}\left(z\left(t_{1}^{-}\right)\right) \rightarrow 0, J_{2}\left(z\left(t_{2}^{-}\right)\right) \rightarrow 0}} e_{2}(t)=\lim _{\substack{J_{1}\left(z\left(t_{1}^{-}\right)\right) \rightarrow 0, J_{2}\left(z\left(t_{2}^{-}\right)\right) \rightarrow 0}}\{z(t)-\widetilde{z}(t)\} \\
& =\frac{1}{\Gamma(q)}\left[z_{a}\left(\ln \frac{t}{a}\right)^{q-1}\right. \\
& +\int_{a}^{t}\left(\ln \frac{t}{s}\right)^{q-1} g(s, z(s)) \frac{d s}{s} \\
& -\left(z_{a}+\int_{a}^{t_{2}} g(s, z(s)) \frac{d s}{s}\right)\left(\ln \frac{t}{t_{2}}\right)^{q-1} \\
& \left.-\int_{t_{2}}^{t}\left(\ln \frac{t}{s}\right)^{q-1} g(s, z(s)) \frac{d s}{s}\right] \text {. }
\end{aligned}
$$

Then, by (25), we obtain

$$
e^{e_{2}(t)}=\frac{\sigma\left[J_{1}\left(z\left(t_{1}^{-}\right)\right)\right]+\sigma\left[J_{2}\left(z\left(t_{2}^{-}\right)\right)\right]-1}{\Gamma(q)}\left[z_{a}\left(\ln \frac{t}{a}\right)^{q-1}\right.
$$

$$
\begin{aligned}
& \left.+\int_{a}^{t}\left(\ln \frac{t}{s}\right)^{q-1} g(s, z(s)) \frac{d s}{s}\right] \\
& +\frac{J_{1}\left(z\left(t_{1}^{-}\right)\right)}{\Gamma(q)}\left(\ln \frac{t}{t_{1}}\right)^{q-1}-\frac{J_{1}\left(z\left(t_{1}^{-}\right)\right)}{\Gamma(q)}\left(\ln \frac{t}{t_{2}}\right)^{q-1} \\
& +\frac{1-\sigma\left[J_{1}\left(z\left(t_{1}^{-}\right)\right)\right]}{\Gamma(q)}\left[\left(z_{a}+\int_{a}^{t_{1}} g(s, z(s)) \frac{d s}{s}\right)\right. \\
& \left.\cdot\left(\ln \frac{t}{t_{1}}\right)^{q-1}+\int_{t_{1}}^{t}\left(\ln \frac{t}{s}\right)^{q-1} g(s, z(s)) \frac{d s}{s}\right] \\
& -\frac{\sigma\left[J_{2}\left(z\left(t_{2}^{-}\right)\right)\right]}{\Gamma(q)}\left[\left(z_{a}+\int_{a}^{t_{2}} g(s, z(s)) \frac{d s}{s}\right)\right. \\
& \left.\cdot\left(\ln \frac{t}{t_{2}}\right)^{q-1}+\int_{t_{2}}^{t}\left(\ln \frac{t}{s}\right)^{q-1} g(s, z(s)) \frac{d s}{s}\right] .
\end{aligned}
$$

Thus,

$$
\begin{aligned}
z(t) & =\widetilde{z}(t)+e_{2}(t) \\
& =\frac{\sigma\left[J_{1}\left(z\left(t_{1}^{-}\right)\right)\right]+\sigma\left[J_{2}\left(z\left(t_{2}^{-}\right)\right)\right]-1}{\Gamma(q)}\left[z_{a}\left(\ln \frac{t}{a}\right)^{q-1}\right. \\
& \left.+\int_{a}^{t}\left(\ln \frac{t}{s}\right)^{q-1} g(s, z(s)) \frac{d s}{s}\right] \\
& +\frac{J_{1}\left(z\left(t_{1}^{-}\right)\right)}{\Gamma(q)}\left(\ln \frac{t}{t_{1}}\right)^{q-1}+\frac{J_{2}\left(z\left(t_{2}^{-}\right)\right)}{\Gamma(q)}\left(\ln \frac{t}{t_{2}}\right)^{q-1} \\
& +\frac{1-\sigma\left[J_{1}\left(z\left(t_{1}^{-}\right)\right)\right]}{\Gamma(q)}\left[\left(z_{a}+\int_{a}^{t_{1}} g(s, z(s)) \frac{d s}{s}\right)\right. \\
& \left.\cdot\left(\ln \frac{t}{t_{1}}\right)^{q-1}+\int_{t_{1}}^{t}\left(\ln \frac{t}{s}\right)^{q-1} g(s, z(s)) \frac{d s}{s}\right] \\
& +\frac{1-\sigma\left[J_{2}\left(z\left(t_{2}^{-}\right)\right)\right]}{\Gamma(q)}\left[\left(z_{a}+\int_{a}^{t_{2}} g(s, z(s)) \frac{d s}{s}\right)\right. \\
& \left.\cdot\left(\ln \frac{t}{t_{2}}\right)^{q-1}+\int_{t_{2}}^{t}\left(\ln \frac{t}{s}\right)^{q-1} g(s, z(s)) \frac{d s}{s}\right]
\end{aligned}
$$


Mathematical Problems in Engineering

7

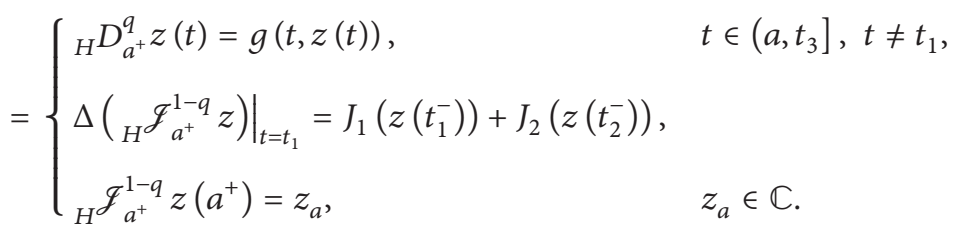

By using (21) and (27) for systems (29) and (28), we obtain

$$
\begin{aligned}
1- & \sigma\left[J_{1}\left(z\left(t_{1}^{-}\right)\right)+J_{2}\left(z\left(t_{2}^{-}\right)\right)\right] \\
& =1-\sigma\left[J_{1}\left(z\left(t_{1}^{-}\right)\right)\right]+1-\sigma\left[J_{2}\left(z\left(t_{2}^{-}\right)\right)\right] .
\end{aligned}
$$

So $1-\sigma(x)=\xi x$ for $\forall x \in \mathbb{C}$, where $\xi$ is an arbitrary constant. Thus, (21) and (27) are rewritten into

$$
\begin{aligned}
& z(t)=\frac{z_{a}}{\Gamma(q)}\left(\ln \frac{t}{a}\right)^{q-1}+\frac{1}{\Gamma(q)} \\
& \cdot \int_{a}^{t}\left(\ln \frac{t}{s}\right)^{q-1} g(s, z(s)) \frac{d s}{s} \\
& +\frac{J_{1}\left(z\left(t_{1}^{-}\right)\right)}{\Gamma(q)}\left(\ln \frac{t}{t_{1}}\right)^{q-1} \\
& -\frac{\xi J_{1}\left(z\left(t_{1}^{-}\right)\right)}{\Gamma(q)}\left\{z_{a}\left(\ln \frac{t}{a}\right)^{q-1}\right. \\
& +\int_{a}^{t}\left(\ln \frac{t}{s}\right)^{q-1} g(s, z(s)) \frac{d s}{s} \\
& -\left[z_{a}+\int_{a}^{t_{1}} g(s, z(s)) \frac{d s}{s}\right]\left(\ln \frac{t}{t_{1}}\right)^{q-1} \\
& \left.-\int_{t_{1}}^{t}\left(\ln \frac{t}{s}\right)^{q-1} g(s, z(s)) \frac{d s}{s}\right\} \quad \text { for } t \in\left(t_{1}, t_{2}\right] \text {, } \\
& z(t)=\frac{z_{a}}{\Gamma(q)}\left(\ln \frac{t}{a}\right)^{q-1}+\frac{1}{\Gamma(q)} \\
& \cdot \int_{a}^{t}\left(\ln \frac{t}{s}\right)^{q-1} g(s, z(s)) \frac{d s}{s} \\
& +\frac{J_{1}\left(z\left(t_{1}^{-}\right)\right)}{\Gamma(q)}\left(\ln \frac{t}{t_{1}}\right)^{q-1}+\frac{J_{2}\left(z\left(t_{2}^{-}\right)\right)}{\Gamma(q)}\left(\ln \frac{t}{t_{2}}\right)^{q-1} \\
& -\frac{\xi J_{1}\left(z\left(t_{1}^{-}\right)\right)}{\Gamma(q)}\left\{z_{a}\left(\ln \frac{t}{a}\right)^{q-1}\right. \\
& +\int_{a}^{t}\left(\ln \frac{t}{s}\right)^{q-1} g(s, z(s)) \frac{d s}{s} \\
& -\left[z_{a}+\int_{a}^{t_{1}} g(s, z(s)) \frac{d s}{s}\right]\left(\ln \frac{t}{t_{1}}\right)^{q-1} \\
& \left.-\int_{t_{1}}^{t}\left(\ln \frac{t}{s}\right)^{q-1} g(s, z(s)) \frac{d s}{s}\right\}
\end{aligned}
$$

$$
\begin{aligned}
& -\frac{\xi J_{2}\left(z\left(t_{2}^{-}\right)\right)}{\Gamma(q)}\left\{z_{a}\left(\ln \frac{t}{a}\right)^{q-1}\right. \\
& +\int_{a}^{t}\left(\ln \frac{t}{s}\right)^{q-1} g(s, z(s)) \frac{d s}{s} \\
& -\left[z_{a}+\int_{a}^{t_{2}} g(s, z(s)) \frac{d s}{s}\right]\left(\ln \frac{t}{t_{2}}\right)^{q-1} \\
& \left.-\int_{t_{2}}^{t}\left(\ln \frac{t}{s}\right)^{q-1} g(s, z(s)) \frac{d s}{s}\right\} \text { for } t \in\left(t_{2}, t_{3}\right] .
\end{aligned}
$$

For $t \in\left(t_{n}, t_{n+1}\right]$, let

$$
\begin{aligned}
& z(t)=\frac{z_{a}}{\Gamma(q)}\left(\ln \frac{t}{a}\right)^{q-1}+\frac{1}{\Gamma(q)} \int_{a}^{t}\left(\ln \frac{t}{s}\right)^{q-1} \\
& \cdot g(s, z(s)) \frac{d s}{s}+\sum_{i=1}^{n} \frac{J_{i}\left(z\left(t_{i}^{-}\right)\right)}{\Gamma(q)}\left(\ln \frac{t}{t_{i}}\right)^{q-1} \\
& -\frac{\xi}{\Gamma(q)} \sum_{i=1}^{n} J_{i}\left(z\left(t_{i}^{-}\right)\right)\left\{z_{a}\left(\ln \frac{t}{a}\right)^{q-1}\right. \\
& +\int_{a}^{t}\left(\ln \frac{t}{s}\right)^{q-1} g(s, z(s)) \frac{d s}{s} \\
& -\left[z_{a}+\int_{a}^{t_{i}} g(s, z(s)) \frac{d s}{s}\right]\left(\ln \frac{t}{t_{i}}\right)^{q-1} \\
& \left.-\int_{t_{i}}^{t}\left(\ln \frac{t}{s}\right)^{q-1} g(s, z(s)) \frac{d s}{s}\right\} \quad \text { for } t \in\left(t_{n}, t_{n+1}\right] .
\end{aligned}
$$

Using (32), we have

$$
\begin{aligned}
H^{\mathcal{J}_{a^{+}}^{1-q} z\left(t_{n+1}^{+}\right)=} & H_{a^{+}}^{\mathcal{J}^{1-q}} z\left(t_{n+1}^{-}\right)+J_{n+1}\left(z\left(t_{n+1}^{-}\right)\right) \\
= & z_{a}+\sum_{i=1}^{n+1} J_{i}\left(z\left(t_{i}^{-}\right)\right) \\
& +\int_{a}^{t_{n+1}} g(s, z(s)) \frac{d s}{s} .
\end{aligned}
$$

Therefore, the approximate solution $\widetilde{z}(t)$ is provided as

$$
\begin{gathered}
\widetilde{z}(t)=\frac{1}{\Gamma(q)}\left({ }_{H} \mathcal{g}_{a^{+}}^{1-q} z\left(t_{n+1}^{+}\right)\right)\left(\ln \frac{t}{t_{n+1}}\right)^{q-1}+\frac{1}{\Gamma(q)} \\
\cdot \int_{t_{n+1}}^{t}\left(\ln \frac{t}{s}\right)^{q-1} g(s, z(s)) \frac{d s}{s}
\end{gathered}
$$


8

Mathematical Problems in Engineering

$$
\begin{aligned}
& =\frac{1}{\Gamma(q)}\left[z_{a}+\sum_{i=1}^{n+1} J_{i}\left(z\left(t_{i}^{-}\right)\right)+\int_{a}^{t_{n+1}} g(s, z(s)) \frac{d s}{s}\right] \\
& \cdot\left(\ln \frac{t}{t_{n+1}}\right)^{q-1}+\frac{1}{\Gamma(q)} \\
& \cdot \int_{t_{n+1}}^{t}\left(\ln \frac{t}{s}\right)^{q-1} g(s, z(s)) \frac{d s}{s}
\end{aligned}
$$

$$
\text { for } t \in\left(t_{n+1}, t_{n+2}\right] \text {. }
$$

Let $e_{n+1}(t)=z(t)-\widetilde{z}(t)$ for $t \in\left(t_{n+1}, t_{n+2}\right]$. On the other hand, by (32), the exact solution $z(t)$ of (1a)-(1c) satisfies

$$
\begin{aligned}
& \lim _{J_{1}\left(z\left(t_{1}^{-}\right)\right) \rightarrow 0, \ldots, J_{n+1}\left(z\left(t_{n+1}^{-}\right)\right) \rightarrow 0} z(t)=\frac{z_{a}}{\Gamma(q)}\left(\ln \frac{t}{a}\right)^{q-1} \\
& +\frac{1}{\Gamma(q)} \int_{a}^{t}\left(\ln \frac{t}{s}\right)^{q-1} g(s, z(s)) \frac{d s}{s} \\
& \text { for } t \in\left(t_{n+1}, t_{n+2}\right] \text {, } \\
& \lim _{\substack{J_{j}\left(z\left(t_{j}^{-}\right)\right) \rightarrow 0, j \in\{1,2, \ldots, n+1\}}} z(t)=\frac{z_{a}}{\Gamma(q)}\left(\ln \frac{t}{a}\right)^{q-1}+\frac{1}{\Gamma(q)} \\
& \cdot \int_{a}^{t}\left(\ln \frac{t}{s}\right)^{q-1} g(s, z(s)) \frac{d s}{s} \\
& +\sum_{\substack{1 \leq i \leq n+1, i \neq j}} \frac{J_{i}\left(z\left(t_{i}^{-}\right)\right)}{\Gamma(q)}\left(\ln \frac{t}{t_{i}}\right)^{q-1} \\
& -\xi \sum_{\substack{1 \leq i \leq n+1, i \neq j}} \frac{J_{i}\left(z\left(t_{i}^{-}\right)\right)}{\Gamma(q)}\left\{z_{a}\left(\ln \frac{t}{a}\right)^{q-1}\right. \\
& +\int_{a}^{t}\left(\ln \frac{t}{s}\right)^{q-1} g(s, z(s)) \frac{d s}{s} \\
& -\left[z_{a}+\int_{a}^{t_{i}} g(s, z(s)) \frac{d s}{s}\right]\left(\ln \frac{t}{t_{i}}\right)^{q-1} \\
& \left.-\int_{t_{i}}^{t}\left(\ln \frac{t}{s}\right)^{q-1} g(s, z(s)) \frac{d s}{s}\right\} \\
& \text { for } t \in\left(t_{n+1}, t_{n+2}\right] \text {. }
\end{aligned}
$$

Therefore,

$$
\begin{gathered}
\lim _{J_{1}\left(z\left(t_{i}^{-}\right)\right) \rightarrow 0,} e_{n+1}(t)=\lim _{J_{1}\left(z\left(t_{i}^{-}\right)\right) \rightarrow 0,}\{z(t)-\tilde{z}(t)\} \\
\vdots \\
J_{n+1}\left(z\left(t_{n+1}^{-}\right)\right) \rightarrow 0 \\
=\frac{1}{\Gamma(q)}\left\{z_{a}\left(\ln \frac{t}{a}\right)^{q-1}+\int_{a}^{t}\left(\ln \frac{t}{s}\right)^{q-1}\right. \\
\left.\cdot g(s, z(s)) \frac{d s}{s}-\left[z_{a}+\int_{a}^{t_{n+1}}\right)\right) \rightarrow 0 \\
\left.g(s, z(s)) \frac{d s}{s}\right]
\end{gathered}
$$

$$
\begin{aligned}
& \left.\cdot\left(\ln \frac{t}{t_{n+1}}\right)^{q-1}-\int_{t_{n+1}}^{t}\left(\ln \frac{t}{s}\right)^{q-1} g(s, z(s)) \frac{d s}{s}\right\}, \\
& \lim _{\substack{J_{j}\left(z\left(t_{j}^{-}\right)\right) \rightarrow 0, j \in\{1,2, \ldots, n+1\}}} e_{n+1}(t)=\lim _{\substack{J_{j}\left(z\left(t_{j}^{-}\right)\right) \rightarrow 0, j \in\{1,2, \ldots, n+1\}}}\{z(t)-\tilde{z}(t)\} \\
& =\frac{1}{\Gamma(q)}\left(1-\xi \sum_{\substack{1 \leq i \leq n+1, i \neq j}} J_{i}\left(z\left(t_{i}^{-}\right)\right)\right)\left[z_{a}\left(\ln \frac{t}{a}\right)^{q-1}\right. \\
& \left.+\int_{a}^{t}\left(\ln \frac{t}{s}\right)^{q-1} g(s, z(s)) \frac{d s}{s}\right] \\
& +\sum_{\substack{1 \leq i \leq n+1, i \neq j}} \frac{J_{i}\left(z\left(t_{i}^{-}\right)\right)}{\Gamma(q)}\left(\ln \frac{t}{t_{i}}\right)^{q-1} \\
& +\xi \sum_{\substack{1 \leq i \leq n+1, i \neq j}} \frac{J_{i}\left(z\left(t_{i}^{-}\right)\right)}{\Gamma(q)}\left\{\left[z_{a}+\int_{a}^{t_{i}} g(s, z(s)) \frac{d s}{s}\right]\right. \\
& \left.\cdot\left(\ln \frac{t}{t_{i}}\right)^{q-1}+\int_{t_{i}}^{t}\left(\ln \frac{t}{s}\right)^{q-1} g(s, z(s)) \frac{d s}{s}\right\} \\
& -\frac{1}{\Gamma(q)}\left(z_{a}+\sum_{\substack{1 \leq i \leq n+1, i \neq j}} J_{i}\left(z\left(t_{i}^{-}\right)\right)\right. \\
& \left.+\int_{a}^{t_{n+1}} g(s, z(s)) \frac{d s}{s}\right)\left(\ln \frac{t}{t_{n+1}}\right)^{q-1}-\frac{1}{\Gamma(q)} \\
& \cdot \int_{t_{n+1}}^{t}\left(\ln \frac{t}{s}\right)^{q-1} g(s, z(s)) \frac{d s}{s} .
\end{aligned}
$$

By (36), we obtain

$$
\begin{aligned}
& e_{n+1}(t)=\frac{1}{\Gamma(q)}\left[1-\xi \sum_{i=1}^{n+1} J_{i}\left(z\left(t_{i}^{-}\right)\right)\right]\left[z_{a}\left(\ln \frac{t}{a}\right)^{q-1}\right. \\
& \left.+\int_{a}^{t}\left(\ln \frac{t}{s}\right)^{q-1} g(s, z(s)) \frac{d s}{s}\right] \\
& +\sum_{i=1}^{n+1} \frac{J_{i}\left(z\left(t_{i}^{-}\right)\right)}{\Gamma(q)}\left(\ln \frac{t}{t_{i}}\right)^{q-1} \\
& +\sum_{i=1}^{n+1} \frac{J_{i}\left(z\left(t_{i}^{-}\right)\right)}{\Gamma(q)}\left(\ln \frac{t}{t_{n+1}}\right)^{q-1} \\
& +\xi \sum_{i=1}^{n+1} \frac{J_{i}\left(z\left(t_{i}^{-}\right)\right)}{\Gamma(q)}\left\{\left[z_{a}+\int_{a}^{t_{i}} g(s, z(s)) \frac{d s}{s}\right]\right.
\end{aligned}
$$




$$
\begin{aligned}
& \left.\cdot\left(\ln \frac{t}{t_{i}}\right)^{q-1}+\int_{t_{i}}^{t}\left(\ln \frac{t}{s}\right)^{q-1} g(s, z(s)) \frac{d s}{s}\right\} \\
& -\frac{1}{\Gamma(q)}\left[z_{a}+\int_{a}^{t_{n+1}} g(s, z(s)) \frac{d s}{s}\right]\left(\ln \frac{t}{t_{n+1}}\right)^{q-1} \\
& -\int_{t_{n+1}}^{t}\left(\ln \frac{t}{s}\right)^{q-1} g(s, z(s)) \frac{d s}{s} \quad \text { for } t \in\left(t_{n+1}, t_{n+2}\right] .
\end{aligned}
$$

Then

$$
\begin{aligned}
& z(t)=\widetilde{z}(t)+e_{n+1}(t)=\frac{z_{a}}{\Gamma(q)}\left(\ln \frac{t}{a}\right)^{q-1}+\frac{1}{\Gamma(q)} \\
& \cdot \int_{a}^{t}\left(\ln \frac{t}{s}\right)^{q-1} g(s, z(s)) \frac{d s}{s} \\
& +\sum_{i=1}^{n+1} \frac{J_{i}\left(z\left(t_{i}^{-}\right)\right)}{\Gamma(q)}\left(\ln \frac{t}{t_{i}}\right)^{q-1}-\frac{\xi}{\Gamma(q)} \sum_{i=1}^{n+1} J_{i}\left(z\left(t_{i}^{-}\right)\right) \\
& \cdot\left\{z_{a}\left(\ln \frac{t}{a}\right)^{q-1}+\int_{a}^{t}\left(\ln \frac{t}{s}\right)^{q-1} g(s, z(s)) \frac{d s}{s}\right. \\
& -\left[z_{a}+\int_{a}^{t_{i}} g(s, z(s)) \frac{d s}{s}\right]\left(\ln \frac{t}{t_{i}}\right)^{q-1}
\end{aligned}
$$

$$
\begin{aligned}
\left.-\int_{t_{i}}^{t}\left(\ln \frac{t}{s}\right)^{q-1} g(s, z(s)) \frac{d s}{s}\right\} & \\
& \text { for } t \in\left(t_{n+1}, t_{n+2}\right] .
\end{aligned}
$$

Thus, the solution of system (1a)-(1c) satisfies (11).

So system (1a)-(1c) is equivalent to (11). The proof is now completed.

\section{An Example}

In this section, an example will be provided to illustrate the usefulness of Theorem 4, which will show that there is general solution for impulsive systems with Hadamard fractional derivatives.

Example. The analytical solution of (1a)-(1c) is difficultly obtained when $f$ is a nonlinear function. Therefore, let us consider an impulsive fractional linear system:

$$
\begin{aligned}
{ }_{H} D_{1^{+}}^{1 / 2} z(t)= & \ln t, \quad t \in(1,3], t \neq 2, \\
\left.\Delta\left({ }_{H^{J_{1}}} \mathcal{F}^{1-1 / 2} z\right)\right|_{t=2}= & { }_{H^{+}} \mathcal{J}_{1^{+}}^{1-1 / 2} z\left(2^{+}\right) \\
& -{ }_{H^{\mathcal{J}_{1}}}^{\mathcal{F}^{1-1 / 2}} z\left(2^{-}\right)=l \in \mathbb{C}, \\
H^{\mathcal{J}_{1^{+}}^{1-1 / 2}} z\left(1^{+}\right)= & z_{1} \in \mathbb{C} .
\end{aligned}
$$

Using Theorem 4, we give general solution of system (39) by

$$
= \begin{cases}\frac{z_{1}}{\Gamma(1 / 2)}(\ln t)^{-1 / 2}+\frac{4}{3} \frac{1}{\Gamma(1 / 2)}(\ln t)^{3 / 2} & \text { for } t \in(1,2], \\ \frac{1}{\Gamma(1 / 2)}\left[\left.z_{1}(\ln t)^{-1 / 2}\right|_{t \geq 1}+\left.\frac{4}{3}(\ln t)^{3 / 2}\right|_{t \geq 1}+\left.l\left(\ln \frac{t}{2}\right)^{-1 / 2}\right|_{t \geq 2}\right] \\ -\frac{\xi l}{\Gamma(1 / 2)}\left[\left.z_{1}(\ln t)^{-1 / 2}\right|_{t \geq 1}+\left.\frac{4}{3}(\ln t)^{3 / 2}\right|_{t \geq 1}-\left.\left(z_{1}+\frac{1}{2} \ln 2\right)\left(\ln \frac{t}{2}\right)^{-1 / 2}\right|_{t \geq 2}-\left.\left(\frac{4}{3}\left(\ln \frac{t}{2}\right)^{3 / 2}+2\left(\ln \frac{t}{2}\right)^{1 / 2} \ln 2\right)\right|_{t \geq 2}\right] \text { for } t \in(2,3],\end{cases}
$$

where $\xi$ is a constant. Next, it will be verified that (40) satisfies all conditions of system (39).

(i) For $t \in(1,2]$ in $(40)$, we have

$$
\left.{ }_{H} D_{1^{+}}^{1 / 2} z(t)\right|_{t \in(1,2]}=\left\{\frac{1}{\Gamma(1-1 / 2)}\left(t \frac{d}{d t}\right)\right.
$$

$$
\begin{aligned}
& \int_{1}^{t}\left(\ln \frac{t}{s}\right)^{1-1 / 2-1}\left[\frac{z_{1}}{\Gamma(1 / 2)}(\ln t)^{-1 / 2}\right. \\
& \left.\left.+\frac{4}{3} \frac{1}{\Gamma(1 / 2)}(\ln t)^{3 / 2}\right] \frac{d s}{s}\right\}_{t \in(1,2]}=\left.\ln t\right|_{t \in(1,2]} .
\end{aligned}
$$




$$
\begin{aligned}
& \left.{ }_{H} D_{1^{+}}^{1 / 2} z(t)\right|_{t \in(2,3]}=\frac{1}{\Gamma(1-1 / 2)}\left(t \frac{d}{d t}\right) \int_{1}^{t}\left(\ln \frac{t}{s}\right)^{1-1 / 2-1}\left\{\frac{1}{\Gamma(1 / 2)}\left[\left.z_{1}(\ln s)^{-1 / 2}\right|_{t \geq 1}+\left.\frac{4}{3}(\ln s)^{3 / 2}\right|_{t \geq 1}+\left.l\left(\ln \frac{s}{2}\right)^{-1 / 2}\right|_{t \geq 2}\right]\right. \\
& \left.-\frac{\xi l}{\Gamma(1 / 2)}\left[\left.z_{1}(\ln s)^{-1 / 2}\right|_{t \geq 1}+\left.\frac{4}{3}(\ln s)^{3 / 2}\right|_{t \geq 1}-\left.\left(z_{1}+\frac{1}{2} \ln ^{2} 2\right)\left(\ln \frac{s}{2}\right)^{-1 / 2}\right|_{t \geq 2}-\left.\left(\frac{4}{3}\left(\ln \frac{s}{2}\right)^{3 / 2}+2\left(\ln \frac{s}{2}\right)^{1 / 2} \ln 2\right)\right|_{t \geq 2}\right]\right\}\left.\frac{d s}{s}\right|_{t \in(2,3]} \\
& =\left.\frac{1}{\Gamma(1-1 / 2) \Gamma(1 / 2)}\left(t \frac{d}{d t}\right) \int_{1}^{t}\left(\ln \frac{t}{s}\right)^{1-1 / 2-1}\left[z_{1}(\ln s)^{-1 / 2}+\frac{4}{3}(\ln s)^{3 / 2}-\xi l z_{1}(\ln s)^{-1 / 2}-\xi l \frac{4}{3}(\ln s)^{3 / 2}\right] \frac{d s}{s}\right|_{t \in(2,3]} \\
& +\frac{l}{\Gamma(1-1 / 2) \Gamma(1 / 2)}\left(t \frac{d}{d t}\right) \int_{2}^{t}\left(\ln \frac{t}{\eta}\right)^{1-1 / 2-1}\left[\left(\ln \frac{\eta}{2}\right)^{-1 / 2}+\xi\left(z_{1}+\frac{1}{2} \ln ^{2} 2\right)\left(\ln \frac{\eta}{2}\right)^{-1 / 2}\right. \\
& \left.+\xi\left(\frac{4}{3}\left(\ln \frac{\eta}{2}\right)^{3 / 2}+2\left(\ln \frac{\eta}{2}\right)^{1 / 2} \ln 2\right)\right]\left.\frac{d \eta}{\eta}\right|_{t \in(2,3]}=\left.(\ln t-\xi l \ln t)_{t \geq 1}\right|_{t \in(2,3]}+\left.(\xi l \ln t)_{t \geq 2}\right|_{t \in(2,3]}=\left.\ln t\right|_{t \in(2,3]} .
\end{aligned}
$$

So (40) satisfies Hadamard fractional derivative of system (39).

By the definition of Hadamard fractional integral, we have

$$
\begin{aligned}
& H^{\mathscr{J}_{1^{+}}^{1-1 / 2}}\left\{\frac { \xi l } { \Gamma ( 1 / 2 ) } \left[\left.z_{1}(\ln t)^{-1 / 2}\right|_{t \geq 1}+\left.\frac{4}{3}(\ln t)^{3 / 2}\right|_{t \geq 1}\right.\right. \\
& -\left.\left(z_{1}+\frac{1}{2} \ln ^{2} 2\right)\left(\ln \frac{t}{2}\right)^{-1 / 2}\right|_{t \geq 2} \\
& \left.\left.-\left.\left(\frac{4}{3}\left(\ln \frac{t}{2}\right)^{3 / 2}+2\left(\ln \frac{t}{2}\right)^{1 / 2} \ln 2\right)\right|_{t \geq 2}\right]\right\}_{t \rightarrow 2^{+}} \\
& =\frac{\xi l}{\Gamma(1 / 2) \Gamma(1 / 2)}\left\{\int _ { 1 } ^ { t } ( \operatorname { l n } \frac { t } { \eta } ) ^ { - 1 / 2 } \left[z_{1}(\ln \eta)^{-1 / 2}\right.\right. \\
& \left.+\frac{4}{3}(\ln \eta)^{3 / 2}\right] \frac{d \eta}{\eta}-\int_{2}^{t}\left(\ln \frac{t}{\eta}\right)^{-1 / 2} \\
& \cdot\left[\left(z_{1}+\frac{1}{2} \ln ^{2} 2\right)\left(\ln \frac{\eta}{2}\right)^{-1 / 2}\right. \\
& \left.\left.+\left(\frac{4}{3}\left(\ln \frac{\eta}{2}\right)^{3 / 2}+2\left(\ln \frac{\eta}{2}\right)^{1 / 2} \ln 2\right)\right] \frac{d \eta}{\eta}\right\}_{t \rightarrow 2^{+}} \\
& =\xi l z_{1}+\left\{\frac{4 \xi l(\ln t-\ln 1)^{2}}{3 \Gamma(1 / 2) \Gamma(1 / 2)} \int_{1}^{t}(1\right. \\
& \left.-\frac{\ln \eta-\ln 1}{\ln t-\ln 1}\right)^{-1 / 2} \\
& \left.\cdot\left(\frac{\ln \eta-\ln 1}{\ln t-\ln 1}\right)^{3 / 2} d \frac{\ln \eta-\ln 1}{\ln t-\ln 1}\right\}_{t \rightarrow 2^{+}}
\end{aligned}
$$

$$
-\xi l\left\{z_{1}+\frac{1}{2} \ln ^{2} 2+\frac{4(\ln t-\ln 2)^{2}}{3 \Gamma(1 / 2) \Gamma(1 / 2)} \int_{2}^{t}(1\right.
$$$$
\left.-\frac{\ln \eta-\ln 2}{\ln t-\ln 2}\right)^{-1 / 2}
$$$$
\cdot\left(\frac{\ln \eta-\ln 2}{\ln t-\ln 2}\right)^{3 / 2} d \frac{\ln \eta-\ln 2}{\ln t-\ln 2}
$$$$
+\frac{2(\ln t-\ln 2)}{\Gamma(1 / 2) \Gamma(1 / 2)} \int_{2}^{t}\left(1-\frac{\ln \eta-\ln 2}{\ln t-\ln 2}\right)^{-1 / 2}
$$$$
\left.\cdot\left(\frac{\ln \eta-\ln 2}{\ln t-\ln 2}\right)^{1 / 2} d \frac{\ln \eta-\ln 2}{\ln t-\ln 2}\right\}_{t \rightarrow 2^{+}}
$$$$
=\xi l z_{1}+\frac{4 \xi l(\ln 2-\ln 1)^{2}}{3 \Gamma(1 / 2) \Gamma(1 / 2)} \frac{\Gamma(1 / 2) \Gamma(5 / 2)}{\Gamma(3)}-\xi l\left[z_{1}\right.
$$$$
\left.+\frac{1}{2} \ln ^{2} 2\right]=0
$$

Therefore,

$$
\begin{aligned}
H^{\mathcal{J}^{1}} & =\left\{\frac{1}{\Gamma(1-1 / 2)} \int_{1}^{t}\left(\ln \frac{t}{\eta}\right)^{1-1 / 2-1} z(\eta) \frac{d \eta}{\eta}\right\}_{t \rightarrow 2^{+}} \\
= & -\left\{\frac{1}{\Gamma(1-1 / 2)} \int_{1}^{\mathcal{J}_{1}^{+}}\left(\ln \frac{t}{\eta}\right)^{1-1 / 2-1} z(\eta) \frac{d \eta}{\eta}\right\}_{t=2^{-}} \\
= & l .
\end{aligned}
$$

That is, (40) satisfies impulsive condition in system (39). 
Finally, for system (39), there exists an implicit condition

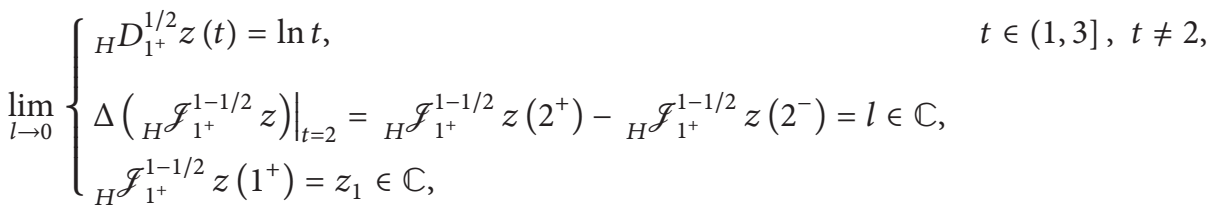

$$
\begin{aligned}
& \longrightarrow\left\{\begin{array}{l}
{ }_{H} D_{1^{+}}^{1 / 2} z(t)=\ln t, \\
{ }_{H}^{J^{1-1 / 2}} z\left(1^{+}\right)=z_{1} \in \mathbb{C} .
\end{array} \quad t \in(1,3],\right.
\end{aligned}
$$

By (40), we have

$$
\lim _{l \rightarrow 0} z(t)= \begin{cases}\frac{z_{1}}{\Gamma(1 / 2)}\left(\int_{1}^{t} \frac{d s}{s}\right)^{1 / 2-1}+\frac{1}{\Gamma(1 / 2)} \int_{1}^{t}\left(\ln \frac{t}{s}\right)^{1 / 2-1} \ln s \frac{d s}{s} \quad \text { for } t \in(1,2] \\ \frac{z_{1}}{\Gamma(1 / 2)}\left(\int_{1}^{t} \frac{d s}{s}\right)^{1 / 2-1}+\frac{1}{\Gamma(1 / 2)} \int_{1}^{t}\left(\ln \frac{t}{s}\right)^{1 / 2-1} \ln s \frac{d s}{s} & \text { for } t \in(2,3]\end{cases}
$$

which shows that (40) satisfies condition (3). So (40) is the general solution of system (39).

\section{Competing Interests}

The authors declare that they have no competing interests.

\section{Acknowledgments}

The work described in this paper is financially supported by the National Natural Science Foundation of China (Grant no. 21576033) and the Natural Science Foundation of Jiangxi Province (Grant no. 20151BAB207013).

\section{References}

[1] I. Podlubny, Fractional Differential Equations, vol. 198 of Mathematics in Science and Engineering, Academic Press, San Diego, Calif, USA, 1999.

[2] A. A. Kilbas, H. H. Srivastava, and J. J. Trujillo, Theory and Applications of Fractional Differential Equations, Elsevier, Amsterdam, The Netherlands, 2006.

[3] D. Baleanu, K. Diethelm, E. Scalas, and J. J. Trujillo, Fractional Calculus Models and Numerical Methods, Series on Complexity, Nonlinearity and Chaos, World Scientific, Singapore, 2012.

[4] H. Ye, J. Gao, and Y. Ding, "A generalized Gronwall inequality and its application to a fractional differential equation," Journal of Mathematical Analysis and Applications, vol. 328, no. 2, pp. 1075-1081, 2007.

[5] M. Benchohra, J. Henderson, S. K. Ntouyas, and A. Ouahab, "Existence results for fractional order functional differential equations with infinite delay," Journal of Mathematical Analysis and Applications, vol. 338, no. 2, pp. 1340-1350, 2008.
[6] R. P. Agarwal, M. Benchohra, and S. Hamani, "A survey on existence results for boundary value problems of nonlinear fractional differential equations and inclusions," Acta Applicandae Mathematicae, vol. 109, no. 3, pp. 973-1033, 2010.

[7] Z. M. Odibat, "Analytic study on linear systems of fractional differential equations," Computers \& Mathematics with Applications, vol. 59, no. 3, pp. 1171-1183, 2010.

[8] B. Ahmad and J. J. Nieto, "Existence of solutions for antiperiodic boundary value problems involving fractional differential equations via Leray-Schauder degree theory," Topological Methods in Nonlinear Analysis, vol. 35, no. 2, pp. 295-304, 2010.

[9] Z. Bai, "On positive solutions of a nonlocal fractional boundary value problem," Nonlinear Analysis: Theory, Methods \& Applications, vol. 72, no. 2, pp. 916-924, 2010.

[10] G. M. Mophou and G. M. N’Guérékata, "Existence of mild solutions of some semilinear neutral fractional functional evolution equations with infinite delay," Applied Mathematics and Computation, vol. 216, no. 1, pp. 61-69, 2010.

[11] W. Deng, "Smoothness and stability of the solutions for nonlinear fractional differential equations," Nonlinear Analysis: Theory, Methods \& Applications, vol. 72, no. 3-4, pp. 1768-1777, 2010.

[12] A. A. Kilbas, "Hadamard-type fractional calculus," Journal of the Korean Mathematical Society, vol. 38, no. 6, pp. 1191-1204, 2001.

[13] P. L. Butzer, A. A. Kilbas, and J. J. Trujillo, "Compositions of Hadamard-type fractional integration operators and the semigroup property," Journal of Mathematical Analysis and Applications, vol. 269, no. 2, pp. 387-400, 2002.

[14] P. L. Butzer, A. A. Kilbas, and J. J. Trujillo, "Mellin transform analysis and integration by parts for Hadamard-type fractional integrals," Journal of Mathematical Analysis and Applications, vol. 270, no. 1, pp. 1-15, 2002.

[15] P. Thiramanus, S. K. Ntouyas, and J. Tariboon, "Existence and uniqueness results for Hadamard-type fractional differential 
equations with nonlocal fractional integral boundary conditions," Abstract and Applied Analysis, vol. 2014, Article ID 902054, 9 pages, 2014.

[16] M. Klimek, "Sequential fractional differential equations with Hadamard derivative," Communications in Nonlinear Science and Numerical Simulation, vol. 16, no. 12, pp. 4689-4697, 2011.

[17] B. Ahmad and S. K. Ntouyas, "A fully Hadamard type integral boundary value problem of a coupled system of fractional differential equations," Fractional Calculus and Applied Analysis, vol. 17, no. 2, pp. 348-360, 2014.

[18] F. Jarad, T. Abdeljawad, and D. Baleanu, "Caputo-type modification of the Hadamard fractional derivatives," Advances in Difference Equations, vol. 2012, article 142, 8 pages, 2012.

[19] Y. Y. Gambo, F. Jarad, D. Baleanu, and T. Abdeljawad, "On Caputo modification of the Hadamard fractional derivatives," Advances in Difference Equations, vol. 2014, article 10, 12 pages, 2014.

[20] Y. Adjabi, F. Jarad, D. Baleanu, and T. Abdeljawad, "On Cauchy problems with Caputo Hadamard fractional derivatives," Journal of Computational Analysis and Applications, vol. 21, no. 4, pp. 661-681, 2016.

[21] B. Ahmad and S. Sivasundaram, "Existence results for nonlinear impulsive hybrid boundary value problems involving fractional differential equations," Nonlinear Analysis: Hybrid Systems, vol. 3, no. 3, pp. 251-258, 2009.

[22] B. Ahmad and S. Sivasundaram, "Existence of solutions for impulsive integral boundary value problems of fractional order," Nonlinear Analysis: Hybrid Systems, vol. 4, no. 1, pp. 134141, 2010.

[23] Y. Tian and Z. Bai, "Existence results for the three-point impulsive boundary value problem involving fractional differential equations," Computer and Mathematics with Applications, vol. 59, no. 8, pp. 2601-2609, 2010.

[24] J. Cao and H. Chen, "Some results on impulsive boundary value problem for fractional differential inclusions," Electronic Journal of Qualitative Theory of Differential Equations, vol. 2011, no. 11, pp. 1-24, 2011.

[25] X. Wang, "Impulsive boundary value problem for nonlinear differential equations of fractional order," Computers \& Mathematics with Applications, vol. 62, no. 5, pp. 2383-2391, 2011.

[26] I. Stamova and G. Stamov, "Stability analysis of impulsive functional systems of fractional order," Communications in Nonlinear Science and Numerical Simulation, vol. 19, no. 3, pp. 702709, 2014.

[27] S. Abbas and M. Benchohra, "Impulsive partial hyperbolic functional differential equations of fractional order with statedependent delay," Fractional Calculus and Applied Analysis, vol. 13, no. 3, pp. 225-244, 2010.

[28] S. Abbas and M. Benchohra, "Upper and lower solutions method for impulsive partial hyperbolic differential equations with fractional order," Nonlinear Analysis: Hybrid Systems, vol. 4, no. 3, pp. 406-413, 2010.

[29] S. Abbas, R. P. Agarwal, and M. Benchohra, "Darboux problem for impulsive partial hyperbolic differential equations of fractional order with variable times and infinite delay," Nonlinear Analysis: Hybrid Systems, vol. 4, no. 4, pp. 818-829, 2010.

[30] S. Abbas, M. Benchohra, and L. Gorniewicz, "Existence theory for impulsive partial hyperbolic functional differential equations involving the Caputo fractional derivative," Scientiae Mathematicae Japonicae, vol. 72, no. 1, pp. 49-60, 2010.
[31] M. Benchohra and D. Seba, "Impulsive partial hyperbolic fractional order differential equations in Banach spaces," Journal of Fractional Calculus and Applications, vol. 1, no. 4, pp. 1-12, 2011.

[32] T. L. Guo and K. Zhang, "Impulsive fractional partial differential equations," Applied Mathematics and Computation, vol. 257, pp. 581-590, 2015.

[33] J. Wang and Y. Zhang, "On the concept and existence of solutions for fractional impulsive systems with Hadamard derivatives," Applied Mathematics Letters, vol. 39, pp. 85-90, 2015.

[34] X. Zhang, X. Zhang, and M. Zhang, "On the concept of general solution for impulsive differential equations of fractional order $q(0,1)$, , Applied Mathematics and Computation, vol. 247, pp. 72$89,2014$.

[35] X. Zhang, "On the concept of general solution for impulsive differential equations of fractional-order $q(1,2)$," Applied Mathematics and Computation, vol. 268, pp. 103-120, 2015.

[36] X. Zhang, "The general solution of differential equations with Caputo-Hadamard fractional derivatives and impulsive effect," Advances in Difference Equations, vol. 2015, article 215, 16 pages, 2015.

[37] X. Zhang, P. Agarwal, Z. Liu, and H. Peng, “The general solution for impulsive differential equations with Riemann-Liouville fractional-order $q \epsilon(1,2)$," Open Mathematics, vol. 13, pp. 908930, 2015.

[38] X. Zhang, T. Shu, H. Cao, Z. Liu, and W. Ding, “The general solution for impulsive differential equations with Hadamard fractional derivative of order $q \epsilon(1,2)$," Advances in Difference Equations, vol. 2016, article 14, 36 pages, 2016.

[39] X. Zhang, X. Zhang, Z. Liu, H. Peng, T. Shu, and S. Yang, "The general solution of impulsive systems with Caputo-Hadamard fractional derivative of order $q \in \mathbb{C}(\Re(q) \in(1,2))$," Mathematical Problems in Engineering, vol. 2016, Article ID 8101802, 20 pages, 2016. 


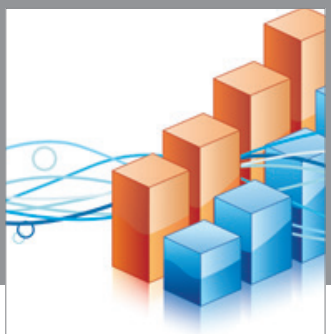

Advances in

Operations Research

vatem alat4

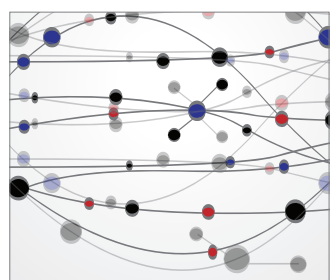

\section{The Scientific} World Journal
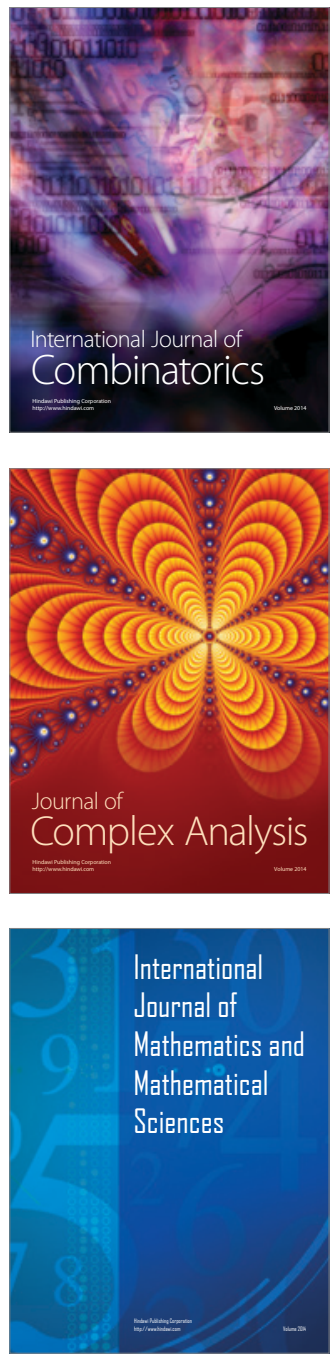
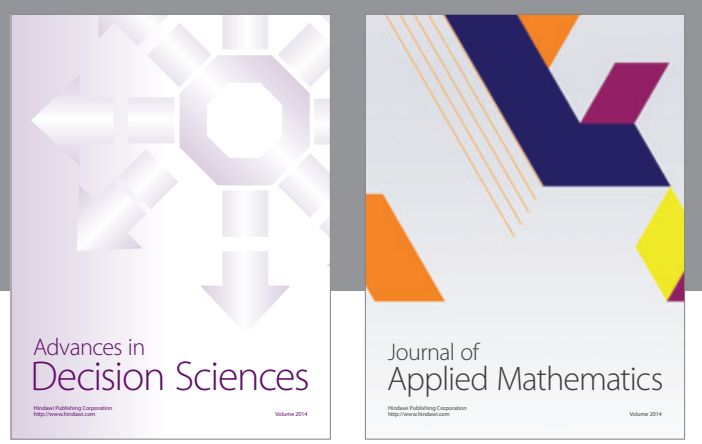

Algebra

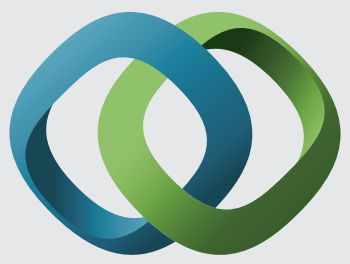

\section{Hindawi}

Submit your manuscripts at

http://www.hindawi.com
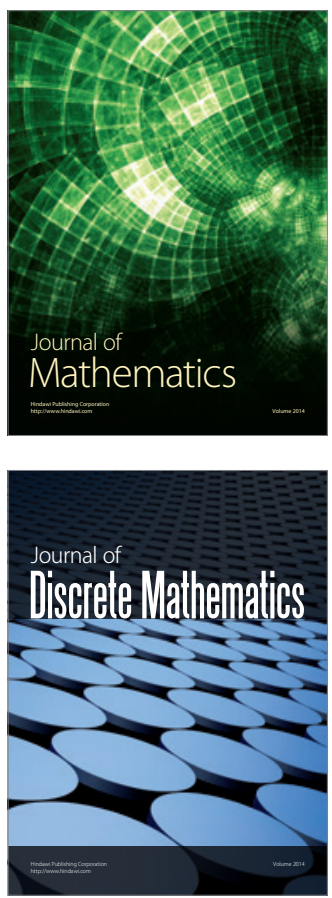

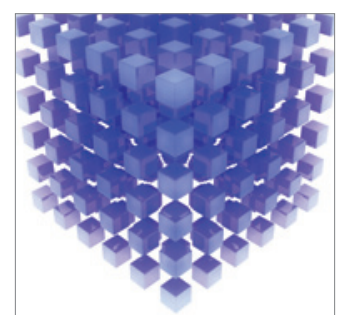

Mathematical Problems in Engineering
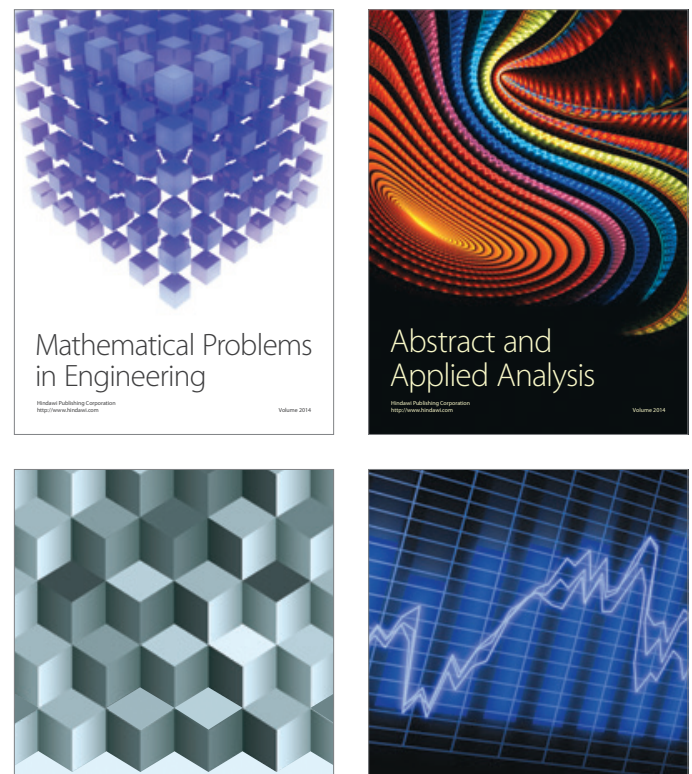

Journal of

Function Spaces

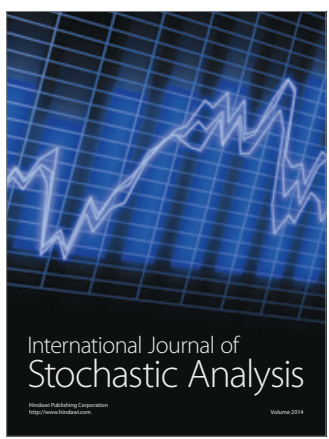

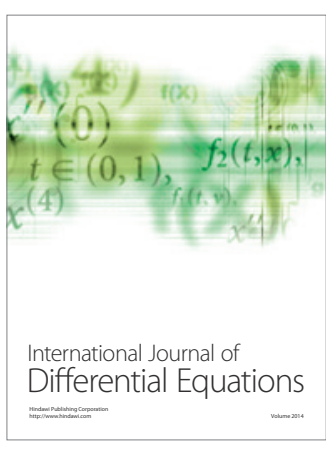
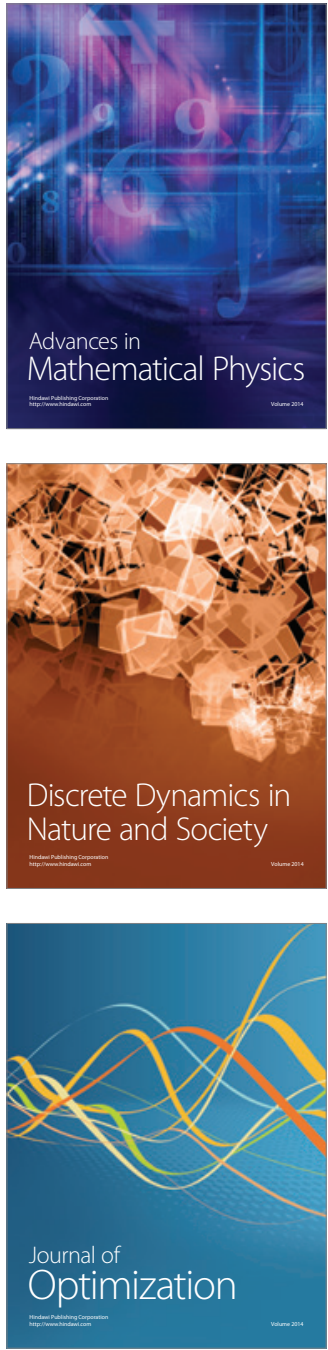